\title{
The benefits and harms of deprescribing
}

\begin{abstract}
Emily Reeve
BPharm(Hons), $\mathrm{PhD}$ Former $\mathrm{PhD}$ candidate ${ }^{1,2}$

Sepehr Shakib MBBS(Hons), FRACP, PhD

Director

Ivanka Hendrix

BPharm, PhD

Clinical Pharmacist ${ }^{3}$

Michael S Roberts

PhD, DSC, FACP

Professor ${ }^{1,4}$

Michael D Wiese

BPharm, MClinPharm, PhD

Senior Lecturer ${ }^{1}$

1 Division of Health Sciences, University of South

Australia, Adelaide, SA.

2 Clinical Pharmacology,

Royal Adelaide Hospital,

Adelaide, SA.

3 Pharmacy Department,

Repatriation General

Hospital, Adelaide, SA.

4 School of Medicine,

University of Queensland,

Brisbane, QLD.

emily.reeve@
\end{abstract}

mymail.unisa.edu.au

doi: 10.5694/mjal3.00200

\section{Reduction in harms associated with polypharmacy}

Being prescribed an increasing number of medications has been associated with increased risk of non-adherence, ADRs, drug-drug and drug-disease interactions, morbidity and mortality. ${ }^{2,3}$ A reduction in the number of medications taken (which may be achieved through identifying and ceasing use of inappropriate medications via a deprescribing process) may theoretically reduce the risk of these negative outcomes. ${ }^{1}$

Pharmacist, medical practitioner and multidisciplinary interventions have all shown effectiveness in reducing polypharmacy, but the scant data on their effects on clinical outcomes are inconsistent. ${ }^{2,3}$ In a recent review of interventions to reduce polypharmacy, only half of the included studies had any outcome measures other than the number of medications taken, and only one-third of those that did measure clinical outcomes showed a benefit. ${ }^{3}$ This may be due to study design limitations, including short follow-up and poor methods of detecting improvements in adverse events. In addition, data supporting the association between polypharmacy and poor outcomes come from epidemiological studies of, at times, thousands of patients; the intervention studies were orders of magnitude smaller and may have been insufficiently powered to detect small improvements in less common events. Therefore, it is still proposed that there is a clinical benefit to reducing polypharmacy through ceasing use of some medications. Alternatively, polypharmacy may simply be a marker for poor overall health, and reducing the number of medications may not alter this trajectory. ${ }^{3}$

\section{Summary}

Deprescribing is the process of trial withdrawal of inappropriate medications.

Currently, the strongest evidence for benefit of deprescribing is from cohort and observational studies of withdrawal of specific medication classes that have shown better patient outcomes, mainly through resolution of adverse drug reactions.

- Additional potential benefits of deprescribing include reduced financial costs and improved adherence with other medications.

The harms of ceasing medication use include adverse drug withdrawal reactions, pharmacokinetic and pharmacodynamic changes and return of the medical condition. These can be minimised with proper planning (ie, tapering), monitoring after withdrawal, and reinitiation of the medication if the condition returns.

More evidence is needed regarding negative, nonreversible effects of ceasing use of certain classes of medication, such as acetylcholinesterase inhibitors.

Cessation of use has not been studied for many medication classes, and large-scale randomised controlled trials of systematic deprescribing are required before the true benefits and harms can be known.

\section{Benefits of ceasing use of inappropriate medications}

When determining the potential benefits of deprescribing, since direct evidence is scant, it may be more relevant to review the evidence related to reduction of IMU (as this is a desired outcome of deprescribing) rather than reduction of polypharmacy. Taking a medication that is inappropriate is, by definition, exposing the patient to more harms than benefits; therefore, ceasing to use this medication will lead to a better overall risk-benefit profile and should, theoretically, lead to better patient outcomes. Again, however, the effect of reduction of IMU on clinical outcomes has not been rigorously studied. ${ }^{4,5} \mathrm{~A}$ systematic review including 24 original studies that investigated interventions to reduce inappropriate prescribing found only one that measured and reported an outcome measure other than appropriateness of medication use. ${ }^{5}$ Other studies that have measured clinical outcomes due to reduced IMU have not shown any effect on ADRs, ${ }^{6}$ or have only shown a non-significant reduction. ${ }^{7}$ Again, limitations in study design, including underpowering, may be responsible for this lack of demonstrated effect. A reduction in drug-drug interactions may also result from deprescribing inappropriate medications, but this has yet to be investigated.

Polypharmacy is not only associated with IMU, but also with underuse of necessary medications, as medical practitioners report a reluctance to start new medications in patients with polypharmacy. ${ }^{8,9}$ Therefore, deprescribing may improve appropriateness of therapy, not just through ceasing use of inappropriate medications, but indirectly via less underuse of appropriate medications. 


\section{Benefits associated with ceasing use of specific classes} of medications

Evidence suggests that withdrawal of specific classes of medications leads to a resolution of ADRs known to be caused by those groups of drugs. For example, discontinuing use of classes of drugs known to increase the risk of falls results in a reduction in falls; discontinuing use of benzodiazepines results in improvement in cognitive and psychomotor abilities; ${ }^{10}$ and withdrawing non-steroidal anti-inflammatory drugs results in improvement in blood pressure. ${ }^{11}$ In addition to such immediate benefits, ceasing use of inappropriate antihypertensives has been shown to be associated with fewer cardiovascular events and a lower mortality rate over a 5 -year follow-up period. ${ }^{12}$ Mortality benefits have also been shown after discontinuing antipsychotic therapy in patients with Alzheimer disease in residential aged care. ${ }^{13}$

Conversely, there are also many studies of medication withdrawal that did not show the expected benefit. ${ }^{10}$ For example, while use of anticholinergic medications in older adults is associated with decreased cognitive function, a randomised controlled trial of withdrawal of these drugs did not show an improvement in cognitive function in patients who were no longer given the medication compared with those who continued to take it. ${ }^{14}$

\section{Other benefits}

The amount of money spent on purchasing medications by Australians and the Australian Government is increasing; ${ }^{15}$ therefore, a potentially significant benefit of deprescribing is a reduction in financial costs associated with medication use. ${ }^{1}$ An Australian study proposed that if the average number of medications taken per person could be reduced by one, an annual cost saving of $\$ 463$ million would result. ${ }^{16}$ Given the high prevalence of IMU, such reductions in medication use, and therefore cost savings, may not be as farfetched as they first seem. Additional cost benefits of deprescribing may be achieved through reduction in ADRs and, consequently, less use of health services.

Deprescribing may improve overall medication adherence through a variety of mechanisms, including reducing the number of medications taken (and costs), simplifying the medication regimen, increasing self-efficacy and reducing ADRs. ${ }^{17}$ Patients report a dislike of taking medications in general and are often uncomfortable with the number of tablets they take, ${ }^{18,19}$ therefore, deprescribing may also lead to improved patient satisfaction.

\section{Potential harms of deprescribing}

\section{Adverse drug withdrawal reactions}

Withdrawal of a medication can result in a physiological response, termed a "withdrawal reaction". 20,21 This can generally be prevented (or minimised) by tapering the dose before withdrawing medication. ${ }^{20}$

A retrospective review of medical records of older adult outpatients revealed that $26 \%$ of occasions of medication cessation led to an adverse withdrawal reaction, and of these, $36 \%$ resulted in increased health service use (eg, hospitalisation or emergency outpatient clinic evaluation). ${ }^{21}$ Similar frequencies have been found among older adults in residential aged care. ${ }^{22}$ While withdrawal reactions can lead to increased health service use, their total impact on health services is likely to be small. A retrospective cohort study showed that withdrawal reactions were the cause of only $1 \%$ of unplanned emergency department visits. This, however, may be an underestimation as the retrospective study design was limited by its reliance on accurate reporting of adverse drug withdrawal reactions. Ceasing use of medications in these patients had not been planned, and hospitalisations may have been prevented if there had been opportunity to taper medications. ${ }^{23}$

\section{Pharmacokinetic and pharmacodynamic changes}

Ceasing use of a medication may result in changing the pharmacokinetics and pharmacodynamics of other medications taken by that patient. For example, discontinuing use of a cytochrome P450 enzyme inhibitor may lead to increased clearance of medications that are metabolised by that enzyme, resulting in decreased serum levels. In patients who are taking both a potassium-lowering and a potassium-increasing medication, ceasing to use one of these has been shown to lead to a change in serum potassium level in most patients and result in hypokalaemia or hyperkalaemia in 3\%-17\%, although the clinical significance of this is unclear. ${ }^{24}$ Currently, very limited work has been done on what surveillance should be conducted when stopping interacting medications, and it is left to the prescriber to determine what follow-up is appropriate on a case-by-case basis.

\section{Return of a medical condition}

Lack of symptoms of a condition may indicate that either the medication is working or that the underlying condition has resolved. It may be appropriate to trial medication withdrawal to determine which of these two scenarios is occurring, and therefore whether the medication was providing a benefit or not. ${ }^{20}$ A systematic review of medication withdrawal trials in older adults showed varying rates of condition relapse for different medications and between studies. If a patient's medical condition returned, restarting the medication resulted in them being quickly resolved. ${ }^{10}$

Of more concern is the potential for discontinuation to negatively and irreversibly affect the medical condition. This is a particular concern with acetylcholinesterase inhibitors (eg, donepezil) in Alzheimer disease. The optimal duration of use of acetylcholinesterase inhibitors is unknown, and although evidence of a benefit beyond 12 months is lacking, the average duration of use is 2 years. This makes these medications potential targets for deprescribing. ${ }^{25}$ Some data, however, have shown that after discontinuing use of donepezil, cognitive scores of patients with mild to moderate dementia dropped below pretreatment baseline levels and did not return to these levels despite reinitiation of donepezil use. ${ }^{26}$ This may have reflected natural disease progression, though, and may be of less concern in patients with severe dementia, among whom donepezil would be targeted for deprescribing. 
A patient-centred deprescribing process*

\begin{tabular}{l|l}
$\begin{array}{l}\text { Step 1: Compile } \\
\text { comprehensive }\end{array}$ & $\begin{array}{l}\text { Obtain an accurate list of all regular, when required and } \\
\text { intermittent medications (prescription and } \\
\text { medication history }\end{array}$ \\
& non-prescription) \\
\hline & - Document indications for each medication \\
- Identify possible ADRs and non-adherence
\end{tabular}

\begin{tabular}{|l|l|}
\hline $\begin{array}{l}\text { Step 2: Identify } \\
\text { potentially } \\
\text { inappropriate } \\
\text { medications }\end{array}$ & $\begin{array}{l}\text { - Assess the potential harms and benefits in the individual } \\
\text { (eg, causing ADRs, no indication) }\end{array}$ \\
& $\begin{array}{l}\text { Use lists of medications that are high risk in the elderly (eg, } \\
\text { Beers list, STOPP criteria) }\end{array}$ \\
& $\begin{array}{l}\text { Take into account life expectancy and the patient's } \\
\text { treatment goals when reviewing preventive medications }\end{array}$ \\
\hline $\begin{array}{l}\text { Step 3: Determine } \\
\text { whether medication } \\
\text { can be ceased, and } \\
\text { prioritise }\end{array}$ & $\begin{array}{l}\text { Appropriate timing of withdrawal (otherwise medically } \\
\text { stable) }\end{array}$ \\
\hline & $\begin{array}{l}\text { Gain patient consent (highlight potential benefits of } \\
\text { medication cessation, confirm that it is a trial and } \\
\end{array}$ \\
\hline
\end{tabular}

\begin{tabular}{|c|c|}
\hline $\begin{array}{l}\text { Step 4: Plan and } \\
\text { initiate withdrawal }\end{array}$ & $\begin{array}{l}\text { Tapering is recommended to reduce adverse drug } \\
\text { withdrawal reactions, increase patient comfort and identify } \\
\text { lowest effective dose } \\
\text { - Ensure the patient (and carer) is comfortable with the plan } \\
\text { and is aware of what steps to take if symptoms return }\end{array}$ \\
\hline $\begin{array}{l}\text { Step 5: Monitor, } \\
\text { support and } \\
\text { document }\end{array}$ & $\begin{array}{l}\text { Monitor the patient for adverse drug withdrawal reactions } \\
\text { and return of symptoms, short and long term } \\
\text { - Ensure that the patient has a contact number for questions } \\
\text { and concerns } \\
\text { - Implement non-pharmacological therapies } \\
\text { - Document the process and outcome and communicate to } \\
\text { all relevant parties }\end{array}$ \\
\hline
\end{tabular}

ADRs = adverse drug reactions. STOPP = Screening Tool Of Older Persons' Potentially Inappropriate Prescriptions. *Adapted from: Reeve E, Shakib S, Hendrix I, et al. ${ }^{30}$

When ceasing use of medications prescribed for a preventive purpose, recurrence of a condition cannot be determined through short-term monitoring of symptoms. Few studies have assessed the safety of withdrawing preventive medications, although long-term monitoring after appropriate withdrawal of antihypertensives and bisphosphonates indicates that it is safe. ${ }^{12,27}$ Deprescribing preventive medications will remove the long-term benefits conferred by their use, potentially increasing mortality. Yet, if the medication is deemed inappropriate and identified for deprescribing then the risks of continuing outweigh the long-term benefits, and stopping their use should, hypothetically, have a net benefit. In older adults, where neither harms nor benefits of many preventive medications are clearly defined, informed decisions about appropriateness (and therefore potential for deprescribing) are, at best, difficult to make. ${ }^{4}$

\section{Conclusions}

Although limited, the evidence to date suggests deprescribing will produce more benefits than harms. ${ }^{28,29}$ Deprescribing can be done safely and may well result in benefits to patients, ${ }^{10}$ although the benefits of deprescribing shown in studies involving specific medication classes may not translate to all medications. Different population groups may experience different harms and benefits from deprescribing. For example, in the palliative care setting the likelihood of achieving a benefit from some medications is lower, as their benefit may not be achieved in the person's remaining life span. However, the benefits of deprescribing may also not be realised, and the stress of making changes to medications and potential withdrawal reactions must also be considered.

Most of the harms of deprescribing can be minimised with proper planning (ie, tapering) and monitoring after use of the drug is discontinued, with reinitiation of the medication if the patient's condition returns. Medications deemed inappropriate (cessation may be recommended because of high levels of harm imposed) may still have some benefits, so loss of benefits cannot be excluded as a harm of deprescribing. More evidence is needed regarding negative, non-reversible effects of ceasing use of certain classes of medication, and to better define the harm-benefit profile of preventive medications in older adults. Additionally there are many medication classes for which cessation of use has not been studied, and large-scale randomised controlled trials of deprescribing according to a predefined protocol are required before the true benefits and harms can be known. We have developed an evidence-based patient-centred deprescribing process that provides practical steps to maximise patient involvement and minimise harms (Box). ${ }^{30}$

Not all patients will ultimately be able to successfully stop taking medications (eg, those with multiple morbidities), and the greatest benefits will be achieved if deprescribing is considered part of a holistic approach to optimising medication use.

Competing interests: Emily Reeve had support from the Australian Government (PhD candidacy funded by the Australian Postgraduate Award) for the submitted work. She received an honorarium for a workshop presentation from the Australian Association of Consultant Pharmacy and payment for work conducted as part of a non-related study, funded by the Society of Hospital Pharmacists of Australia Celgene Information Technology in Hospital Pharmacy grant, in the previous 3 years.

Provenance: Not commissioned; externally peer reviewed.

1 Woodward MC. Deprescribing: achieving better health outcomes for older people through reducing medications. J Pharm Pract Res 2003; 33: 323-328.

2 Hajjar ER, Cafiero AC, Hanlon JT. Polypharmacy in elderly patients. Am J Geriatr Pharmacother 2007; 5: 345-351.

3 Gnjidic D, Le Couteur DG, Kouladjian L, Hilmer SN. Deprescribing trials: methods to reduce polypharmacy and the impact on prescribing and clinical outcomes. Clin Geriatr Med 2012; 28: 237-253.

4 Spinewine A, Schmader KE, Barber N, et al. Appropriate prescribing in elderly people: how well can it be measured and optimised? Lancet 2007; 370: 173-184.

5 Kaur S, Mitchell G, Vitetta L, Roberts MS. Interventions that can reduce inappropriate prescribing in the elderly: a systematic review. Drugs Aging 2009; 26: 1013-1028.

6 Gurwitz JH, Field TS, Rochon P, et al. Effect of computerized provider order entry with clinical decision support on adverse drug events in the long-term care setting. J Am Geriatr Soc 2008; 56: 2225-2233.

7 Hanlon JT, Weinberger M, Samsa GP, et al. A randomized, controlled trial of a clinical pharmacist intervention to improve inappropriate prescribing in elderly outpatients with polypharmacy. Am J Med 1996; 100: 428-437.

8 Mansur N, Weiss A, Beloosesky Y. Relationship of in-hospital medication modifications of elderly patients to postdischarge medications, adherence, and mortality. Ann Pharmacother 2008; 42: 783-789.

9 Fuat A, Hungin AP, Murphy JJ. Barriers to accurate diagnosis and effective management of heart failure in primary care: qualitative study. BMJ 2003; 326: 196.

10 Iyer S, Naganathan V, McLachlan AJ, Le Couteur DG. Medication withdrawal trials in people aged 65 years and older: a systematic review. Drugs Aging 2008; 25: 1021-1031.

11 McKellar GE, Hampson R, Tierney A, et al. Nonsteroidal antiinflammatory drug withdrawal in patients with stable rheumatoid arthritis. J Rheumato 2011; 38: 2150-2152. 
12 Ekbom T, Lindholm LH, Odén A, et al. A 5-year prospective, observational study of the withdrawal of antihypertensive treatment in elderly people. J Intern Med 1994; 235: 581-588.

13 Ballard C, Hanney ML, Theodoulou M, et al. The dementia antipsychotic withdrawal trial (DART-AD): long-term follow-up of a randomised placebo-controlled trial. Lancet Neurol 2009; 8: 151-157.

14 Kersten $\mathrm{H}$, Molden E, Tolo IK, et al. Cognitive effects of reducing anticholinergic drug burden in a frail elderly population: a randomized controlled trial. J Gerontol A Biol Sci Med Sci 2013; 68: 271-278.

15 Kemp A, Preen DB, Glover J, et al. How much do we spend on prescription medicines? Out-of-pocket costs for patients in Australia and other OECD countries. Aust Health Rev 2011; 35: 341-349.

16 Eckert KA, Shi Z, Taylor AW, et al. Learning from an epidemiological, population-based study on prescribed medicine use in adults. Pharmacoepidemiol Drug Saf 2013; 22: 271-277.

17 Reeve E, Wiese MD. Benefits of deprescribing on patients' adherence to medications. Int J Clin Pharm 2014; 36: 26-29.

18 Reeve E, Wiese MD, Hendrix I, et al. People's attitudes, beliefs, and experiences regarding polypharmacy and willingness to deprescribe. J Am Geriatr Soc 2013; 61: 1508-1514.

19 Reeve E, To J, Hendrix I, et al. Patient barriers to and enablers of deprescribing: a systematic review. Drugs Aging 2013; 30: 793-807.

20 Bain KT, Holmes HM, Beers MH, et al. Discontinuing medications: a novel approach for revising the prescribing stage of the medication-use process. J Am Geriatr Soc 2008; 56: 1946-1952.

21 Graves T, Hanlon JT, Schmader KE, et al. Adverse events after discontinuing medications in elderly outpatients. Arch Intern Med 1997; 157: 2205-2210.
22 Gerety MB, Cornell JE, Plichta DT, Eimer M. Adverse events related to drugs and drug withdrawal in nursing home residents. J Am Geriatr Soc 1993; 41: 1326-1332.

23 Marcum ZA, Pugh MJV, Amuan ME, et al. Prevalence of potentially preventable unplanned hospitalizations caused by therapeutic failures and adverse drug withdrawal events among older veterans. J Gerontol $A$ Biol Sci Med Sci 2012; 67: 867-874.

24 Uijtendaal EV, Zwart-van Rijkom JE, van Solinge WW, Egberts TC. Serum potassium influencing interacting drugs: risk-modifying strategies also needed at discontinuation. Ann Pharmacother 2012; 46: 176-182.

25 Parsons C, Hughes CM, Passmore AP, Lapane KL. Withholding, discontinuing and withdrawing medications in dementia patients at the end of life: a neglected problem in the disadvantaged dying? Drugs Aging 2010; 27: 435-449.

26 Doody RS, Geldmacher DS, Gordon B, et al. Open-label, multicenter, phase 3 extension study of the safety and efficacy of donepezil in patients with Alzheimer disease. Arch Neurol 2001; 58: 427-433.

27 Black DM, Schwartz AV, Ensrud KE, et al. Effects of continuing or stopping alendronate after 5 years of treatment: the Fracture Intervention Trial Long-term Extension (FLEX): a randomized trial. JAMA 2006; 296: 2927-2938

28 Garfinkel D, Mangin D. Feasibility study of a systematic approach for discontinuation of multiple medications in older adults: addressing polypharmacy. Arch Intern Med 2010; 170: 1648-1654.

29 Beer C, Loh Pk, Peng YG, et al. A pilot randomized controlled trial of deprescribing. Ther Adv Drug Saf 2011; 2: 37-43.

30 Reeve E, Shakib S, Hendrix I, et al. Review of deprescribing processes and development of an evidence based, patient-centred deprescribing process. Brit J Clin Pharm 2014; Mar 25. [Epub ahead of print]. doi: 10.111/ bcp.12386. 